\title{
Atopische Erkrankungen \\ Neurodermitis als multifaktorielle Dispositionskrankheit
}

\author{
A. Kreuter, P. Altmeyer, Dermatologische Klinik der Ruhr-Universität Bochum, Bochum \\ (Direktor: Prof. Dr. med. P. Altmeyer)
}

NOTFALLMEDIZIN 2003, 29: 236-237

Das atopische Ekzem ist eine der häufigsten dermatologischen Erkrankungen. Der multifaktoriellen Dispositionskrankheit scheint eine genetisch determinierte Überempfindlichkeit gegenüber Umweltfaktoren in Assoziation mit einer erhöhten IgE-Bildung sowie einer veränderten unspezifischen Reaktivität zugrunde zu liegen. Neben einer konsequenten Allergenkarenz umfasst die Basistherapie des atopischen Ekzems den Einsatz von rückfettenden, blanden Externa und Ölbädern. Bei akuten ekzematösen Hautveränderungen können potente topische Glukokortikosteroide kurzfristig eingesetzt werden. Bei längerer Anwendung sollten Kortikoide der Wirkklasse 1 und 2 bevorzugt werden. Ein festes Standbein der Therapie des atopischen Ekzems ist die Phototherapie: Neben der UVA-UVB-Kombinationstherapie und der PUVATherapie hat sich besonders die UVA1-Kaltlichttherapie bewährt.
D as atopische Ekzem (Neurodermitis, atopische Dermatitis) ist eine chronisch-rezidivierende, entzündliche Hauterkrankung, die durch meist stark juckende, erythematöse oder papulöse Hautveränderungen gekennzeichnet ist. Das atopische Ekzem ist eine der häufigsten dermatologischen Erkrankungen. Etwa 15 Millionen Menschen sind in den Industrienationen betroffen. Das atopische Ekzem gehört, wie die Rhinoconjunctivitis allergica und das allergische Asthma bronchiale, zum Formenkreis „Atopie“. Der multifaktoriellen Dispositionskrankheit scheint eine genetisch determinierte Überempfindlichkeit gegenüber Umweltfaktoren unterschiedlichster Art in Assoziation mit einer erhöhten IgE-Bildung sowie einer veränderten unspezifischen Reaktivität zugrunde zu liegen. Nicht selten manifestiert sich das atopisches Ekzem bereits im Kleinkindesalter (Milchschorf). Klinisch charakteristisch für den Verlauf des atopischen Ekzems sind Beugenekzeme (Abb. 1) mit Vergröberung des Hautfaltenreliefs (Lichenifikation) sowie Kratzexkoriationen (Abb. 2). Krankheitsschübe können durch Klima, Jahreszeit, Allergenexposition, Infektionen oder psychische Faktoren ausgelöst werden. Patienten mit atopischem Ekzem sind durch die gestörte Hautbarrierefunktion besonders anfällig für bakterielle und virale Hautinfektionen. So können Infektionen mit Staphylococcus aureus zu großflächigen, erosiv nässenden, impetigoartigen Verkrustungen führen. Beim Eczema herpeticatum handelt es sich um eine großflächige Herpes-simplex-VirusInfektion durch Auto- oder Heteroinokulation. Mollusca contagiosa 
(Dellwarzen) sind besonders häufig bei Kindern mit atopischem Ekzem zu finden.

\section{- Therapie}

Neben einer konsequenten Allergenkarenz (sowohl Aeroallergene als auch Nahrungsmittel) umfasst die Basistherapie des atopischen Ekzems den Einsatz von rückfettenden, blanden Externa und Ölbädern. Die trockene, empfindliche Haut des Atopikers entsteht durch die gestörte Barrierefunktion des Stratum corneum. Als Basistherapeutika werden Emulsionen vom Typ Öl in Wasser (Hydrolotio, hydrophile Creme) und Wasser in Öl (Lipolotio, lipohile Creme) eingesetzt. Der Einsatz von Harnstoff hat eine zusätzlich hydratisierende Wirkung. Bei akuten ekzematösen Hautveränderungen können potente topische Glukokortikosteroide kurzfristig eingesetzt werden. Bei längerer Anwendung sollten Kortikoide der Wirkklasse 1 und 2 (z.B. Hydrokortison) bevorzugt werden. Die Einführung der neuen, nicht halogenisierten Kortikoide mit deutlich verbessertem Nutzen-Risikoverhältnis hat einen entscheidenden Fortschritt in der Lokaltherapie des atopischen Ekzems gebracht. Seit dem Jahre 2002 ist der topische Immunmodulator FK506 (Tacrolimus), ein isoliertes Macrolid-Lakton, für die Behandlung des atopischen Ekzems zugelassen. Lokal appliziert hemmt es die Transkription verschiedener Zytokingene sowie die IgE-induzierte Freisetzung von Histamin. Tacrolimus ist besonders im Gesichtsbereich, wo sich lokale Glukokortikosteroide verbieten, eine neue Behandlungsoption. Ein festes Standbein der Therapie des atopischen Ekzems ist die Phototherapie. Neben der UVA-UVB-Kombinationstherapie und der PUVA-Therapie hat sich besonders die UVA1-Kaltlichttherapie in der Behandlung des atopischen Ekzems bewährt. Auch Schmalspektrum-UVB (311 $\mathrm{nm})$ kann eingesetzt werden. Systemische Glukokortikosteroide sollten nur bei schwerster, exazerbierter, atopischer Dermatitis kurzzeitig zum Einsatz kommen. Auf den Einsatz systemischer Immunsuppressiva (z.B. Methotrexat, Ciclosporin)

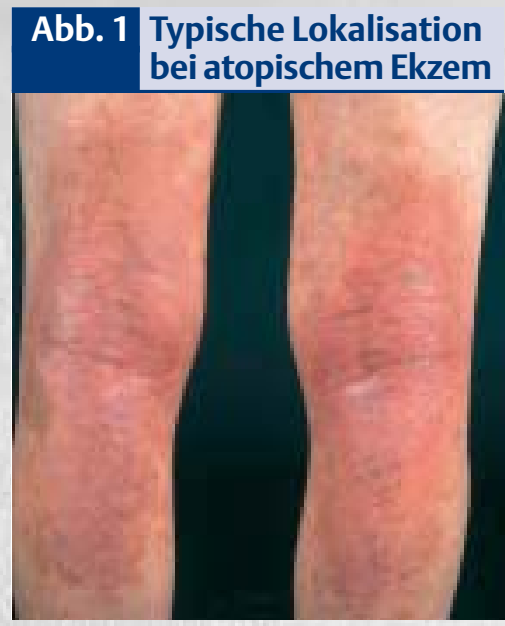

muss gegebenenfalls bei Therapierefraktären, chronisch aktiven Formen zurückgegriffen werden.

Ein differenziertes Verständnis des atopischen Ekzems, einer multifaktoriellen Dispositionskrankheit, ist wesentliche Voraussetzung für die Entwicklung neuer, effizienter Therapiestrategien.

\section{Summary}

Atopic eczema is one of the most common dermatological disorders. This multifactorial disease appears to be based on genetically determined hypersensitivity vis-à-vis environmental factors in association with an elevated production of $\operatorname{IgE}$ and alteredunspecific reactivity. In addition to the rigorous avoidance all allergens, the basic treatment of atopic eczema includes the use of bland fat-replenishing topical agents and oil baths. In the case of acute eczematous skin disorders, glucocorticoids may be applied over the short term. If used over longer periods, class 1 and 2 corticoids should be given preference. An established pillar in the treatment of atopic eczema is phototherapy: in addition to combination therapy with UVA-UVB and PUVA therapy, UVA1 cold light treatment has proven to be of value.

\section{Literatur}

1. Bigby M. A thorough systematic review of treatments for atopic eczema. Arch Dermatol. 2001; 137: 1635-1636

2. Brehler R, Hildebrand A, Luger TA. Recent developments in the treatment of atopic eczema. J Am Acad Dermatol. 1997; 36: 983-994

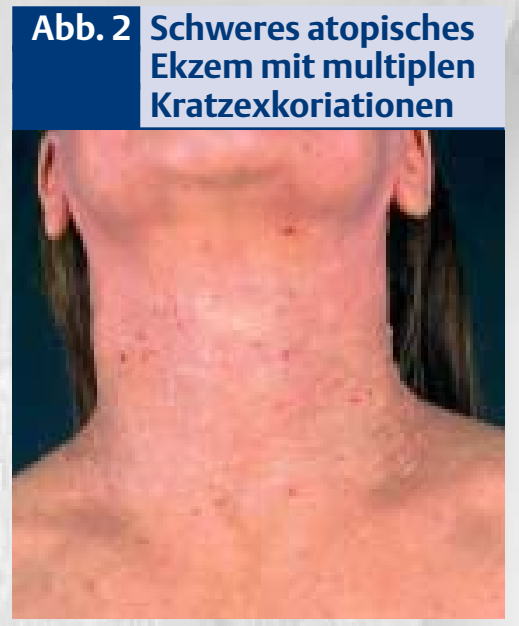

3. Furue M, Terao H, Rikihisa W et al. Clinical dose and adverse effects of topical steroids in daily management of atopic dermatitis. Br J Dermatol. 2003; 148: 128-133

4. Gewirtz AT, Sitaraman SV. Tacrolimus fujisawa. Curr Opin Investig Drugs. 2002; 3: 1307-1311

5. Hoare C, Li Wan Po A, Williams H. Systematic review of treatments for atopic eczema. Health Technol Assess. 2000; 4: 1-191 6. Leung DY, Bieber T. Atopic dermatitis. Lancet. 2003; 361: 151-160

7. Ong CS. Tacrolimus ointment for atopic dermatitis. N Engl J Med. 1998; 339: 1788-1789

8. Ravenscroft JC, Thomas KS, Williams HC. Current management of atopic eczema. Practitioner. 2002; 246: 690-695

9. Reitamo S, Remitz A, Kyllonen H, Saarikko J, Granlund H. Topical noncorticosteroid immunomodulation in the treatment of atopic dermatitis. Am J Clin Dermatol. 2002; 3: 381-388

10. Reynolds NJ, Franklin V, Gray JC et al. Narrow-band ultraviolet $B$ and broad-band ultraviolet $A$ phototherapy in adult atopic eczema: a randomised controlled trial. Lancet. 2001; 357: 2012-2016

11. Russell JJ. Topical tacrolimus: a new therapy for atopic dermatitis. Am Fam Physician. 2002; 6: 1899-1902

12. Tajima T, Ibe M, Matsushita T, Kamide R. A variety of skin responses to ultraviolet irradiation in patients with atopic dermatitis. J Dermatol Sci. 1998; 17: 101-107

13. Thestrup-Pedersen K. Treatment principles of atopic dermatitis. J Eur Acad Dermatol Venereol. 2002; 16: 1-9

14. Wellington K, Jarvis B. Topical pimecrolimus: a review of its clinical potential in the management of atopic dermatitis. Drugs. 2002; 62: 817-840

15. Worm M. Novel therapies for atopic eczema. Curr Opin Investig Drugs. 2002; 3 : 1596-1603

\section{Anschrift für die Verfasser}

Dr. med. Alexander Kreuter

Dermatologische Klinik der Ruhr-Universität Bochum

Gudrunstraße 56

44791 Bochum 